\title{
PARTO VAGINAL EN GESTANTES CON CESÁREA PREVIA EN UN HOSPITAL GENERAL DE PERÚ
}

\author{
VAGINAL BIRTH IN PREGNANT CESAREA IN A GENERAL HOSPITAL OF PERU
}

George Ybaseta-Soto $^{1, a}$, Carmen Quijandria-Tataje ${ }^{2, b}$, Marjorie Ybaseta-Soto ${ }^{3, b}$.

1. Hospital Santa María del Socorro. Ica, Perú.

2. Hospital Augusto Hernández Mendoza IV Es Salud. Ica, Perú.

3. Universidad Privada San Juan Bautista Filial Ica. Ica, Perú.

a. Bachiller en Medicina Humana

b. Ginecólogo Obstetra

DOI: https://doi.org/10.35563/rmp.v9i1.290

\section{Correspondencia:}

George Ybaseta Soto

Dirección: Urb. Villa del Médico B-

7, Ica, Perú.

Teléfono: (+51) 999613922

Correo electrónico:

georgeginoybaseta@outlook.com

Contribuciones De Autoría:

GYS ha participado en la

concepción y diseño del artículo, recolección de los datos. COT y

MYS participaron en el análisis estadístico de datos. Todos los autores participaron en la

interpretación de los datos, redacción del manuscrito, revisión crítica del manuscrito y aprobaron la versión final.

Conflicto De Intereses: Los autores declaran no tener conflictos de interés. Financiamiento: Autofinanciado

\section{Como Citar}

Ybaseta-Soto G. Quijandría Tataje C. Ybaseta-Soto M. Parto vaginal en gestantes con cesárea previa en un Hospital General de Perú. Rev méd panacea. 2020; 9(1): 4-12. doi: https://doi.org/10.35563/rmp.v9i1.290

Recibido: 20 - 11 - 2019

Aceptado: 06 - 02 - 2020

Publicado: 04 - 03 - 2020

\section{RESUMEN}

Objetivo: Determinar el perfil clínico y las principales complicaciones maternas perinatales del parto vaginal en gestantes con cesárea previas en el Hospital Santa María del Socorro Ica, en el año 2018. Materiales y métodos: Se realizó un estudio retrospectivo, descriptivo, observacional. Se revisó las historias clínicas de pacientes con embarazo a término normo evolutivo y antecedente de cesárea en alguna gestación previa, que se sometieron a trabajo de parto y culminaron su parto por vía vaginal o abdominal y que fueron atendidas en el Hospital Santa María del Socorro de lca en el año 2018, se utilizó una ficha de recolección de Datos. Resultados: Se analizaron los datos de 162 gestantes en trabajo de parto, 128 (79\%) lograron el nacimiento por vía vaginal, mientras que 34 (21\%) tuvieron su finalización del embarazo por vía abdominal. Entre las características sociodemográficas predominó: Las edades entre los 20 y 30 años $(80 \%)$, el grado de instrucción secundaria (62\%), estado civil soltera (41\%), ocupación empleada (44\%) y de procedencia rural $(81 \%$. En relación a las complicaciones maternas: la hemorragia post parto estuvo presente en ambos grupos $5,4 \%$ en parto vaginal y $18 \%$ en parto abdominal, las diferencias fueron significativas $(p<0,05)$. Respecto al factor perinatal: la patología predominante fue síndrome de distrés respiratorio, igualmente la diferencia estadística fue significativa $(p<0,05)$. Conclusiones: Las complicaciones maternas en pacientes con parto vaginal o abdominal con antecedentes de cesárea fueron hemorragia post parto y síndrome de distrés respiratorio respectivamente.

Palabras clave: Trabajo de parto, cesárea, Apgar. (Fuente: DeCS BIREME).

\section{ABSTRACT}

Objective: To determine the clinical profile and the main maternal perinatal complications of vaginal birth in pregnant women with previous caesarean section at the Hospital Santa María del Socorro Ica, in 2018. Materials and methods: A retrospective, descriptive, observational study was carried out. The medical records of patients with a normal evolutionary term pregnancy and a history of caesarean section in a previous pregnancy were reviewed. Ica in the year 2018, you will see a data collection sheet. Results: Data from 162 pregnant women in labor were analyzed, 128 (79\%) achieved vaginal birth, while 34 (21\%) had their pregnancy terminated by abdominal route. Among the sociodemographic characteristics predominated: The ages between 20 and 30 years $(80 \%)$, the degree of secondary education (62\%), single marital status $(41 \%)$, employed occupation (44\%) and of rural origin ( $81 \%$ In relation to maternal complications: postpartum hemorrhage was present in both groups $5.4 \%$ in vaginal delivery and $18 \%$ in abdominal delivery, the differences were severe $(p<0.05)$. Regarding the perinatal factor: the pathology predominantly it was respiratory distress syndrome, the statistical difference was also significantly significant $(p<0.05)$. Conclusions: Maternal complications in patients with vaginal or abdominal delivery with a history of caesarean section were postpartum hemorrhage and respiratory distress syndrome respectively. Keywords: Labor obstetric, cesarean section, Apgar score (source: MeSH NLM). 


\section{INTRODUCCIÓN}

El aumento de las tasas de cesárea en muchos países durante los últimos años ha constituido un problema de salud pública, el procedimiento, aunque común, conlleva a un mayor riesgo de morbilidad y mortalidad materna $(1,2)$. La controversia en cuanto a la interrogante de la vía del nacimiento en embarazos de mujeres con antecedente de cesárea es de actualidad, así mismo existe la necesidad de tener más resultados que incentiven la práctica del parto vaginal en cesárea previa.

Desde los años ochenta se han publicado muchos trabajos en los que se muestran las ventajas del parto vaginal después de una cesárea previa, en cuanto a la reducción de costes y morbilidad materna, sin empeoramiento del pronóstico fetal $(2,6)$. Los avances en el manejo del parto en mujeres con una cesárea previa han inclinado a los gineco-obstetras a permitir el trabajo del parto a pacientes con antecedentes de dos cicatrices uterinas $(2,3,4)$.

En el mundo, la incidencia de cesáreas varían y puede oscilar entre el $15 \%$ y el $40 \%$, en Estados Unidos se reporta incremento notable en la tasa de cesárea desde $5.5 \%$ en 1965 a 27\% en 1997, en Latinoamérica, Chile ostenta la tasa más alta de cesáreas con $40 \%$ y Haití con menos de $15 \%$ posee la tasa más baja, sin embargo en el caso del último se encuentra también que más del $20 \%$ de su población no recibe atención del parto. Según la OMS la tasa de cesáreas debería encontrarse idealmente entre 10 - $15 \%$, sin embargo esta tasa en el mundogeneralmente no desciende de $20 \%(2,5)$.

En el Perú, la tasa de cesáreas varía de acuerdo al establecimiento de Salud, existiendo hospitales con tasas de cesárea de $54 \%$, siendo la causa más frecuente el hecho de haberse sometido a cesárea en el parto anterior $(6,7)$.

Existen informes estadísticos de principales Hospitales maternos perinatales donde se consignan tasas no menores al $20 \%$ tanto del Ministerio de Salud y ESSALUD, Los Hospitales de referencia como la Maternidad de Lima y Edgardo Rebagliatti que varían entre 20 a 38\%, a diferencia del Hospital San Bartolomé donde estudios realizados reportan una incidencia de 18\%(6-8).

Según diversas investigaciones de distintos continentes se puede decir que someter a una prueba de trabajo de parto a pacientes con antecedentes de cesárea cuando los factores del parto vaginal son favorables esto logra un incremento de partos vaginales sin mayor complicación $(2,9,10)$.

Actualmente los Gineco-obstetras saben que toda gestante que ha tenido un parto por cesárea puede tener lo que se llama parto vaginal después de un parto por cesárea (PVDC). EI PVDC conlleva ciertos riesgos pero ofrece ventajas, al decidir si el PVDC se debe considerar el factor clave de la causa de la cesárea previa, factores obstétricos o ciertos padecimientos médicos durante el embarazo que sean causa de contraindicación $(2,11)$.

La prueba de trabajo de parto después de una cesárea tiene una probabilidad de éxito entre 65 y $80 \%$ con escasa complicación materna o neonatal. En los última década los ginecólogos se han abocado a esta tarea de dar oportunidad a los pacientes con cesárea previa a tener parto vaginal cuidando las posibles complicaciones (12).

"De por si la vía natural ofrece ventajas tanto para la madre como para el feto, relacionadas con una menor morbilidad, evolución más fisiológica, menor incidencia de infecciones, menor necesidad de transfusiones sanguíneas y menor estadía hospitalaria, lo que mejora el establecimiento del vínculo madre-recién nacido" (11).

Actualmente en nuestro medio no se cuenta con uniformidad de criterios y/o factores de riesgo que nos permitan dilucidar la vía de culminación de la presente gestación de pacientes con una cesárea previa. Se busca investigar si resulta beneficioso la inducción y/o estimulación del trabajo de parto en las gestantes con el antecedente de cesárea anterior. Asimismo existen pocos estudios respecto a la estancia hospitalaria de las puérperas sometidas a parto vaginal sea espontáneo o inducido respecto de las cesareadas con criterios de electivas, así como de sus neonatos.

En tal sentido esta investigación responde al objetivo dar resultados sobre el perfil clínico y las principales complicaciones materno perinatal de las pacientes gestantes con antecedente de cesárea que se sometieron a trabajo de parto y la vía de culminación de la misma en el año 2018 en el Hospital Santa María del Socorro de Ica, resultados que proponen que el parto vaginal sea una alternativa segura tanto para la madre como para el feto, así mismo favorecerá la reducción de la tasa de cesáreas con la consecuente disminución de los costos en la atención de la mujer gestante.

\section{MATERIALES Y MÉTODOS}

Es un estudio descriptivo, retrospectivo de tipo comparativo, efectuado en base a 2200 historias clínicas perinatales almacenadas en el servicio de estadística del Hospital Santa María del Socorro de Ica, Perú, desde el 01 de enero al 31 de diciembre del 2018.

El tamaño de la muestra estuvo conformada por el total de las gestantes (162) que con antecedente de cesárea que cumplieron con los criterios de estudio registrados en la base de datos del HSMS de los cuales habían culminado su gestación por vía vaginal (128 gestantes) y cesárea (34 gestantes). 
Para el estudio se ha tomado en su mayoría los criterios de la ACOG $(2,24)$.

Criterios de inclusión: presentación cefálica, gestación única a término, cicatriz obstétrica segmentaria, ausencia de malformación uterina, confrontación cefalopélvica favorable, localización normal de la placenta, ausencia de patología materna o fetal diagnosticada antes del inicio de trabajo de parto, gestantes con cesárea previa que finalizaron su gestación en el Hospital Santa María del Socorro de Ica e historia clínica con datos completos.

Criterios de Exclusión: gestantes que continúan con el diagnóstico anterior, antecedente de cesárea corporal clásica, de incisión uterina en "T" invertida, de Incisión uterina vertical inferior, cirugía previa con acceso a cavidad, presentaciones o situaciones fetales anómalas, historia clínica no disponible y/o con información requerida incompleta y periodo intergenésico corto.

De los casos elegibles se recolectó la información en una ficha especialmente diseñada para este estudio, considerando como variables: edad materna, tipo de parto previo, edad gestacional, patología obstétrica asociada, dilatación al ingreso, acentuación del trabajo de parto, número de controles prenatales, periodo intergenésico, peso del recién nacido, APGAR, complicaciones maternas, grado de instrucción y estado civil.

Los pacientes que ingresan al estudio están registrados en el libro de hospitalización de Emergencia de GinecoObstetricia del HSMSI

De la base de datos informático y de las historias clínicas en físico se procedió a recoger la información necesaria para la elaboración del presente trabajo, en concordancia con las variables, mediante la aplicación de una ficha confeccionada especialmente para el estudio.

Se tomó de las Historias Clínicas los datos referentes al objetivo del trabajo y al contenido de la ficha de recolección de datos, se tiene toda la reserva del caso protegiendo la confidencialidad de los pacientes.

Los datos se recolectaron en el programa Excel. Para todas las variables se determinará las frecuencias y porcentajes. Para evaluar diferencias de las variables analizadas según tipo de parto se utilizó la prueba estadística de Chi cuadrado, considerándose significativo si $\mathrm{P}<0.05$. No se hizo cálculo de la muestra por cuanto se incluyó a toda la población con criterios de inclusión.

Se realizaron las gestiones para que Dirección de investigación de la Facultad de Medicina Humana de la Universidad San Luis Gonzaga de Ica autorice la ejecución de la investigación, también el Comité Institucional de Ética en Investigación de la institución donde se realizó la investigación aprobó el proyecto y otorgue el permiso correspondiente para realizar esta investigación.

Tabla1. Características sociodemográficas de las pacientes en trabajo de parto con cesárea previa en el hospital Santa María del Socorro de Ica, Perú, 2018.

\begin{tabular}{|c|c|c|c|c|c|c|}
\hline \multicolumn{7}{|c|}{ Tipo de parto } \\
\hline \multirow[t]{2}{*}{ Caracteris as Generales } & \multicolumn{2}{|c|}{ Grupo de parto vaginal } & \multicolumn{2}{|c|}{ Grupo de parto por cesárea } & \multirow[b]{2}{*}{ Chi 2} & \multirow[b]{2}{*}{$\mathbf{p}$} \\
\hline & $\mathbf{n}$ & $\%$ & $\mathbf{n}$ & $\%$ & & \\
\hline \multicolumn{7}{|l|}{ Edad } \\
\hline$<19$ años & 8 & 6 & 5 & 15 & \multirow{4}{*}{36,59} & \multirow{4}{*}{$<0,05$} \\
\hline 20 a 30 años & 102 & 80 & 18 & 53 & & \\
\hline 30 a 35 años & 10 & 8 & 7 & 21 & & \\
\hline$>35$ años & 8 & 6 & 4 & 11 & & \\
\hline \multicolumn{7}{|l|}{ Grado de instrucción } \\
\hline Primaria & 40 & 32 & 18 & 53 & \multirow{3}{*}{4,79} & \multirow{3}{*}{$<0,05$} \\
\hline Secundaria & 80 & 62 & 9 & 26 & & \\
\hline Superior & 8 & 6 & 7 & 21 & & \\
\hline \multicolumn{7}{|l|}{ Estado civil } \\
\hline Soltera & 52 & 41 & 10 & 29 & \multirow{3}{*}{0,03} & \multirow{3}{*}{$>0,05$} \\
\hline Casada & 28 & 22 & 8 & 24 & & \\
\hline Conviviente & 48 & 37 & 16 & 47 & & \\
\hline \multicolumn{7}{|l|}{ Ocupación } \\
\hline Ama de casa & 44 & 34 & 8 & 24 & \multirow{4}{*}{0,16} & \multirow{4}{*}{$>0,05$} \\
\hline Empleada & 56 & 44 & 17 & טכ & & \\
\hline Independiente & 25 & 20 & 8 & 24 & & \\
\hline Otros & 3 & 2 & 1 & 2 & & \\
\hline \multicolumn{7}{|l|}{ Domicilio } \\
\hline Rural & 104 & 81 & 6 & 18 & \multirow{2}{*}{12,6} & \multirow{2}{*}{$<0,05$} \\
\hline Urbano & 24 & 19 & 28 & 82 & & \\
\hline
\end{tabular}




\section{RESULTADOS}

La población estudiada estuvo conformada por el total de las gestantes con antecedente de cesárea sin contraindicaciones para parto por la vía natural, que fueron sometidas a trabajo de parto, siendo un total de 162 pacientes de los cuales 128 (79\%) gestantes tuvieron parto vaginal y 34 (21\%) pacientes terminaron su gestación por vía alta o abdominal.

En la tabla 1, respecto a las características sociodemográficas de pacientes en trabajo de parto con cesárea previa se observa que las características más predominantes en ambos grupos de estudio fueron el grupo etario de 20 a 30 años 102 (80\%) para parto vaginal y 18 (53\%) para parto por cesárea, en cuanto al grado de instrucción predominó los del estudio secundario 80 (62\%) para parto vaginal y 9 (26\%) para parto por cesárea, en cuanto al estado civil fue el grupo de las solteras $52(41 \%)$ y $10(29 \%)$ tanto para el parto vaginal como para la cesárea, en cuanto a la ocupación el grupo predominante fueron los empleados en ambos grupos 56 (44\%) y 17 (50\%) respectivamente y en relación al domicilio predominó el grupo que vivía en zona rural $104(81 \%)$ y $6(18 \%)$ tanto para parto vaginal como para cesárea respectivamente.

Se halló diferencias significativas $(p<0,05)$ de la edad, grado de instrucción y domicilio según los grupos analizados.

Tabla 2. Características sociodemográficas de las pacientes en trabajo de parto con cesárea previa en el hospital Santa María del Socorro de Ica, Perú, 2018.

\begin{tabular}{|c|c|c|c|c|c|c|}
\hline \multicolumn{7}{|c|}{ Tipo de parto } \\
\hline \multirow[t]{2}{*}{ Caracteris as Generales } & \multicolumn{2}{|c|}{ Grupo de parto vaginal } & \multicolumn{2}{|c|}{ Grupo de parto por cesárea } & \multirow[b]{2}{*}{ Chi 2} & \multirow[b]{2}{*}{$p$} \\
\hline & $\mathbf{n}$ & $\%$ & $\mathbf{n}$ & $\%$ & & \\
\hline \multicolumn{7}{|l|}{ Paridad } \\
\hline Primípara & 72 & 72 & 28 & 28 & \multirow{2}{*}{7,75} & \multirow{2}{*}{$<0,05$} \\
\hline Mulpara & 56 & 90 & 6 & 10 & & \\
\hline \multicolumn{7}{|l|}{ Control parental } \\
\hline Inadecuada & 54 & 68 & 26 & 32 & \multirow{2}{*}{12,63} & \multirow{2}{*}{$<0,05$} \\
\hline Adecuada & 74 & 90 & 8 & 10 & & \\
\hline \multicolumn{7}{|l|}{ Periodo intergenesico } \\
\hline 2 a 4 años & 80 & 80 & 20 & $<u$ & \multirow[t]{2}{*}{0,15} & \multirow[t]{2}{*}{$>0,05$} \\
\hline > a 4 años & 48 & 77 & 14 & 23 & & \\
\hline \multicolumn{7}{|l|}{ Edad gestacional } \\
\hline$<40$ semanas & 108 & 83 & 22 & 11 & \multirow[t]{2}{*}{6,56} & \multirow[t]{2}{*}{$<0,05$} \\
\hline$>40$ semanas & 20 & 63 & 12 & 37 & & \\
\hline
\end{tabular}

En la Tabla 2, se presenta sobre las características obstétricas de las pacientes en trabajo de parto con cesárea previa se observó en la característica paridad que el grupo predominante fue las primíparas 72 (72\%) y 28 (28\%) tanto para las pacientes del parto vaginal y parto cesárea respectivamente.

Para la característica control prenatal predomino el grupo con un CPN inadecuado 54(68\%) y 26 (32\%) tanto para el grupo parto vaginal y cesárea respectivamente.

Para la característica Periodo Intergenésico predominó el grupo con periodo intergenésico de 2 a 4 años 80 (80\%) y 20 (20\%) tanto para el parto vaginal y parto cesárea respectivamente y para la edad gestacional predominó la edad gestacional menor de 40 semanas en ambos grupos.

Se halló diferencias significativas $(p<0,05)$ de la paridad, control prenatal y edad gestacional en los grupos analizados.

Tabla 3. Parto vaginal con cesárea previa en el hospital Santa María del Socorro de Ica, Perú, 2018. Según causa de cesárea.

\begin{tabular}{lccccc}
\multicolumn{1}{c}{ Causa de cesárea } & Grupo de parto vaginal & Grupo departo por cesárea & \\
& $n$ & $\%$ & $n$ & $\%$ & Chi 2 \\
Preeclampsia & 6 & 22 & 21 & 78 & $0>0,05$ \\
Trabajo de parto disfunc. & 5 & 33 & 10 & 77 & $0,01>0,05$ \\
Sufrimiento fetal & 2 & 66 & 1 & 34 & $0,013>0,05$ \\
Desproporción cefalopelvico & 3 & 100 & 0 & 0 & \\
Distocia de situación & 1 & 50 & 1 & 50 & \\
Presentación podálica & 2 & 100 & 0 & 0 & \\
Distocia variedad de posición & 5 & 83 & 1 & 17 & \\
\hline
\end{tabular}


En la tabla 3, sobre las causas de la cesárea, se observa que las causas más frecuentes de cesárea fueron: preeclampsia (78 \%) y trabajo de parto disfuncional (77\%). En este caso no se halló diferencias significativas según las variables analizadas.

Tabla 4. Parto vaginal con cesárea previa en el hospital Santa María del Socorro de Ica, Perú, 2018 según complicaciones obstétricas.

\begin{tabular}{|c|c|c|c|c|c|c|}
\hline \multicolumn{7}{|c|}{ Tipo de parto } \\
\hline \multirow[t]{2}{*}{ Complicaciones obstétricas } & \multicolumn{2}{|c|}{ Grupo de parto vaginal } & \multicolumn{2}{|c|}{ Grupo de parto por cesárea } & \multirow[b]{2}{*}{ Chi 2} & \multirow[b]{2}{*}{$\mathbf{p}$} \\
\hline & $\mathbf{n}$ & $\%$ & $\mathbf{n}$ & $\%$ & & \\
\hline Edema vulvar & 10 & 90 & 1 & 10 & & \\
\hline Desgarros & 5 & 100 & 0 & 0 & & \\
\hline Hematoma & 7 & 100 & 0 & 0 & & \\
\hline Hemorragia post parto & 7 & 53 & 6 & 47 & & \\
\hline Atonía uterina & 4 & 80 & 1 & 20 & 5,31 & $<0,05$ \\
\hline Ruptura uterina & 0 & 0 & 0 & 0 & & \\
\hline Infección de herida operada & 1 & 33 & 2 & 64 & & \\
\hline Histerectomía & 0 & 0 & 0 & 0 & & \\
\hline Muerte materna & 0 & 0 & 0 & 0 & & \\
\hline
\end{tabular}

Tabla 4, se observa que la complicación mayor más importante fue la hemorragia post parto 7 (53\%) y 6 (57\%) cifras similares tanto para el parto vaginal y parto por cesárea, seguido por la atonía uterina 4 (80\%) y 1 (20\%) tanto para el parto vaginal y cesárea.

Al comparar las complicaciones obstétricas, se halló diferencias significativas $(p<0,05)$ de la complicación hemorragia post parto en los grupos analizados.

Tabla 5. Parto vaginal después de una cesárea, en pacientes atendidas en el hospital Santa María del Socorro de Ica, Perú, 2018 según peso y apgar a los 5 minutos

\begin{tabular}{|c|c|c|c|c|c|c|}
\hline \multicolumn{7}{|c|}{ Tipo de parto } \\
\hline \multirow[t]{2}{*}{ Peso y apgardel R.N. } & \multicolumn{2}{|c|}{ Grupo de parto vaginal } & \multicolumn{2}{|c|}{ Grupo de parto porcesárea } & \multirow[b]{2}{*}{ Chi 2} & \multirow[b]{2}{*}{$\mathbf{p}$} \\
\hline & $\mathbf{n}$ & $\%$ & $\mathbf{n}$ & $\%$ & & \\
\hline \multicolumn{7}{|l|}{ Peso } \\
\hline$<2500$ gramos & 1 & 25 & 3 & 75 & & \\
\hline 2500 a 3999 gramos & 127 & 84 & 24 & 16 & 13,51 & $<0,05$ \\
\hline$\geq 4000$ gramos & 0 & 0 & 1 & 100 & & \\
\hline \multicolumn{7}{|l|}{ Apgar } \\
\hline$\leq 3$ & 0 & 0 & 0 & 0 & & \\
\hline 4 a 6 & 1 & 50 & 1 & 50 & 1,03 & $>0,05$ \\
\hline$\geq 7$ & 127 & 80 & 33 & 20 & & \\
\hline
\end{tabular}

En la tabla 5, con respecto al peso y apgar para el grupo de PVDC, al evaluar el peso del R.N., la mayor frecuencia se observa en el grupo de peso de 2500 a 3999 siendo 127 (84\%) que tuvieron parto vaginal y al evaluar el Apgar el grupo de mayor predominio fue los R.N. que adquirieron puntaje mayor o igual a 7 grupo que nacieron por vía vaginal.

Se halló diferencias significativas $(p<0,05)$ del peso de 2500 a 3999 gramos, en los grupos analizados 
Tabla 6. Parto vaginal con cesárea previa, hospital Santa María del Socorro de Ica, Perú, 2018 según complicaciones perinatales.

\begin{tabular}{lcccccc} 
& \multicolumn{7}{c}{ Tipo de parto } & & & \\
Complicación perinatal & Grupo de parto vaginal & Grupo de parto por cesárea & & \\
& $n$ & $\%$ & $n$ & $\%$ & Chi 2 & p \\
Asfixia perinatal & 4 & 100 & 0 & 0 & 0,07 & $>0,05$ \\
Trauma obstétrico & 2 & 100 & 0 & 0 & & \\
SDR & 3 & 33 & 6 & 64 & 11,99 & $<0,05$ \\
Ingreso UCIN & 1 & 20 & 4 & 80 & & \\
Muerte neonatal & 0 & 0 & 0 & 0 & & \\
Ninguno & 118 & 83 & 24 & 17 & & \\
\hline
\end{tabular}

SDR= Síndrome de distrés respiratorio

En la Tabla 6, en relación a las complicaciones perinatales, se observa que la mayor frecuencia de complicación fueron asfixia perinatal 4(100\%), trauma obstétrico 2(100\%) y SDR 3(33\%). se halló diferencia estadísticamente significativa p< 0,05 para SDR.

\section{DISCUSIÒN}

El Colegio Americano de Obstetras y Ginecólogos desde 1981 fomenta la prueba de parto vaginal en gestantes con cesárea anterior, además de haber diversos autores que indican que del 65 al $80 \%$ de todas ellas culminan en un parto vaginal $(7,9)$ sin mínimo riesgo de complicaciones, resultados que son parecidos al hallado en este estudio.

La alta probabilidad de éxito del PVDC evita el alto costo social y económico que conlleva el efectuar una nueva cesárea, disminuyendo los días de hospitalización y la morbilidad puerperal o complicaciones tanto para la madre como para el recién nacido (13).

En cuanto a las características sociodemográficas de las pacientes en estudio, estas se encuentra distribuidas a predominio de: gestantes entre las edades de 20 a 30 años, de pacientes con educación secundaria, solteras y convivientes, de ocupación empleadas y en el grupo de procedencia rural. Se estableció relación estadísticamente significativa en las variables edad, grado de instrucción y procedencia rural y el éxito del parto vaginal $(p<0,05)$.

En la Tabla 1, se determina que el grupo etario predominante fue el de 20 a 30 años con un éxito para PVDC del $80 \%$, Algert y col., y otros autores hallan resultados similares (14), Bujold et al., publicaron que en mujeres de 35 años o más el porcentaje de parto vaginal fue del $65,1 \%$, frente al $70,7 \%$ en mujeres de $30-34$ años y el $71,9 \%$ en mujeres menores de 30 años, disminuyendo las tasas de éxito mientras aumentaba la edad (15).

El grueso de pacientes en estudio se encuentran en el grupo de edad reproductiva más sobresaliente y probablemente sus condiciones fisiológicas propias dela edad han tenido algún tipo de efecto favorable en el mecanismo del trabajo de parto, tal como se puede establecer la revisión de las bases teóricas y científicas siendo mucho más favorable el parto en mujeres jóvenes.

Los resultados en cuanto al estado civil son semejantes a lo encontrado por Espino Felipa E., en el Hospital María Auxiliadora en el año 2016 (16).

En cuanto al grado de Instrucción y el PVDC la mayor parte se encuentra distribuida en el grupo de educación secundaria y primaria para el parto vaginal. Siendo un grupo menos frecuente el de educación superior lo que colisiona con el hecho de que tener un grado de conocimiento respecto al cuidado de su salud podría ayudar al éxito del PVDC.

Se esperaría que un mayor grado de instrucción permitiera a las gestantes una mejor comprensión de los riesgos y beneficios de someterse al parto vaginal, de tal modo que la escolaridad podría influir en la toma de decisión de la paciente en la vía final de parto pues es un factor favorable en la prevención de las patologías y el riesgo de la salud, en el estudio no resulta influyente esta versión para el éxito del PVDC.

En el estudio se observa que existe un predominio de madres convivientes y solteras haciendo un total de $78 \%$, sin embargo el estado civil de la madre en nuestro estudio no tiene un valor estadísticamente significativo, aunque sería muy conveniente realizar otros estudios para poder estar mucho más seguros de tal sospecha de que tendría relación con el éxito de PVDC. 
En cuanto a la procedencia el PVDC fue a predominio del grupo rural lo que puede estar en relación al control prenatal adecuado y la importancia para estas mujeres del regreso temprano a sus labores.

En la tabla 2, la variable paridad es estadísticamente significativa, se evidencia una mayor frecuencia de PVDC en primíparas probablemente ha tenido relación con otros factores clínicos favorables para el éxito de PVDC.

Con respecto a los controles prenatales (Tabla 2), el periodo intergenesico mayor a dos años y la edad gestacional menor a 40 semanas tuvieron una frecuencia predominante, características asociadas al PVDC resultados que guardan relación con los encontrados por Bujold y cols., quien encontró que un período intergenésico menor o igual a 24 meses se asoció a casi 3 veces la probabilidad de rotura uterina (17).

El PVDC tuvo éxito en el $80 \%$ de los casos en gestaciones menores 40 semanas resultado similar encontrado por Gil Clares D. (18) que encontró que en el grupo de pacientes con cesárea previa con gestaciones menores a 40 semanas tenían un éxito de $82,9 \%$ para PVDC, mientras que Marca Ticona D. no encontró asociación estadística entre la edad gestacional menor a 40 semanas y PVDC (19), sin embargo diverso autores refieren que la edad gestacional menor a 40 semanas es un factor positivo para el PVDC $(18,19)$.

La causa más frecuente de cesárea actual es la preeclampsia con (78\%), seguido por trabajo de parto disfuncional (77\%) y sufrimiento fetal (34\%) (Tabla 3), causas que son similares a lo informado por Gil Clares D. en su estudio realizado en el Hospital Augusto Hernández de Essalud de Ica en el 2014, donde se seleccionaron las pacientes con antecedentes de cesárea, siendo los motivos más frecuentes de indicación de cesárea urgente: trabajo de parto disfuncional $(41,9 \%)$, sufrimiento fetal $12,9 \%$, pero difiere en y riesgo de pérdida de bienestar fetal $(13,5 \%)(18)$, sin embargo difiere la causa actual menos frecuente fue la preeclamsia disto a lo encontrado en nuestro estudio donde fue la primera causa que podría estar en relación en que se manejan poblaciones socio demográficamente distintas

En la tabla 4, al evaluar las complicaciones mayores la única variable que tiene significancia estadística es la hemorragia post parto siendo la probabilidad por igual en ambos grupos. Al respecto, esta complicación también fue encontrada por Leal Mateos M. y col. en su estudio donde reporta a la hemorragia posparto como una de las complicaciones importantes, en su estudio reporta que el porcentaje de complicaciones maternas fue significativamente mayor en el grupo de pacientes a las que se les sometió a prueba de trabajo de parto y necesitaron una cesárea intraparto, con un 18\% (20).

Cabe señalar que los resultados reportados en los diversos estudios, respecto a las complicaciones maternas no son uniformes y en algunos se evidencia un mayor porcentaje y riesgo de complicaciones en las gestantes sometidas a prueba de trabajo de parto lo cual dificulta la unificación de criterios para el manejo de estos casos.

La principal preocupación en torno al parto vaginal en pacientes con cesárea previa, son las complicaciones por rotura uterina y hemorragia por atonía uterina que necesite histerectomía o que de estas puedan provocar muerte materna, afortunadamente no hubo ningún caso $(17,21,22,23,24)$.

En la tabla 5 , se muestra que el $84 \%$ de recién nacidos tienen un peso dentro de los percentiles normales diversos autores encuentran que un peso menor a 4000 gramos favorece el éxito del parto vaginal, Elkousy y cols., refiere que cuando el peso es de 4000 grs tienen un éxito reducido para PVDC o mas existe riesgo de rotura uterina $(22,23)$.

En cuanto al estado de los RN que ingresaron al estudio (Tabla 7), no hubo fallecidos, es más el $80 \%$ de RN tuvieron un APGAR mayor o igual a 7 a los 5 minutos congruente con datos de Lindo Pérez $\mathrm{M}$ Et al., quien obtuvo en su estudio una tasa de éxito del 54,7\% PVDC de donde el 99,4\% de los RN tuvieron un APGAR mayor o igual a 7 a los 5 minutos concluye que el parto vaginal en una gestante con antecedente de cesárea se considera seguro, con altas probabilidades de éxito para el bienestar del recién nacido. $(23,24)$.

En el estudio solo se encontró morbilidad perinatal con diferencia estadística para el SDR siendo en mayor porcentaje en el grupo de cesáreas. Por lo general el pronóstico perinatal al igual que otros estudios es favorable para el PVDC $(1,2,23,25)$.

\section{CONCLUSIÓN}

El PVDC sin contraindicaciones se considera con altas probabilidades de éxito materno perinatal y mínimo riesgo de complicaciones mayores, sin embargo debe existir un control obstétrico estricto para intervenir principalmente antes de la rotura uterina que podría convertirse en una emergencia que atente contra la vida de la madre y el producto de la gestación. 


\section{REFERENCIAS BIBLIOGRÁFICAS}

1. Arrunátegui Quispe Grecia. Factores predictores para prueba de parto vaginal exitosa en gestantes con cesárea previa en el Hospital de Especialidades Básicas la Noria. (Tesis Para optar el Título profesional de Médico Cirujano) Trujillo-Perú. Universidad Privada Antenor Orrego 2015.

2. Carlos Quesnel García-Benítez, Miguel de Jesús López-Rioja, Diana Elena Monzalbo-Núñez. Parto después de cesárea ¿una opción segura? Ginecol Obstet Mex2015; 83:69-87 http://www.medigraphic.com/pdfs/ginobsmex/gom-2 015/gom152b.pdf

3. Mejía LC. Prevalencia de cesáreas en gestantes atendidas en el Hospital Es salud II-Cañete durante el periodo de enero a diciembre del 2014. (Tesis Para optar el Título profesional de Licenciado en Obstetricia) Lima-Perú. Universidad Ricardo Palma 2016.

http://repositorio.urp.edu.pe/handle/urp/556

4. Ceriani Cernadas, José M. et al. Nacimiento por cesárea al término en embarazos de bajo riesgo: efectos sobre la morbilidad neonatal. Arch. argent. pediatr., Buenos Aires, 2010; 108(1): 23-33 Disponible en

<http://www.scielo.org.ar/scielo.php?script=sci_arttext \&pid=S0325-00752010000100005\&lng=es\&nrm =iso $>$. accedido en 21 nov. 2015.

5. Ye J, Betran AP, Vela MG, Souza JP, Zhang J. Searching for the Optimal Rate of Medically Necessary Cesarean Delivery. Birth. 2014;41(3):237-43. https://onlinelibrary.wiley.com/doi/abs/10.1111/birt.12 104

6. Bobadilla Ubillús Lucía Elena, León Jimenez Franco. En torno a las cesáreas en el Perú: ¿solo un problema de cifras?. Rev. peru. ginecol. obstet. [Internet]. 2017 Oct [citado 2019 Mar 12] ; 63( 4 ): 659-660. Disponible en: http://www.scielo.org.pe/scielo.php?script=sci_arttext \&pid=S2304- 51322017000400022\&lng=es.

7. Cuero-Vidal, Olga lucía; Clavijo-prado, Carlos Andrés. Parto vaginal después de una cesárea previa, Hospital San Juan de Dios, Cali (Colombia). Estudio de cohorte. Revista Colombiana de Obstetricia y Ginecología. 2015;62(2): 148-154.

https://revista.fecolsog.org/index.php/rcog/article/vie w/231

8. Alcántara Pérez Manuel. Parto vaginal en la cesareada anterior una vez en el Hospital Docente Madre-Niño San Bartolomé durante el año 2000-2002. Tesis para Optar el Grado de Especialista en Gineco-Obstetricia. UNMSM. Lima -Perú. 2005. http://cybertesis.unmsm.edu.pe/bitstream/handle/cyb ertesis/1855/Perez_ap.pdf?sequence $=1$

9. Vargas T, Lévano J, Lazo M, Parto Vaginal después de una cesárea, aplicando un puntaje al momento del ingreso en un Hospital. Rev. Peruana de Ginecología y Obstetricia [Internet] 2013[Fecha de consulta: 2016];59: 261-266.URL disponible en:

http://sisbib.unmsm.edu.pe/BVRevistas/ginecologia/v ol59_n4/pdf/a05v59n4.pdf2.

10. Sáenz C, Santana S, Torres L, Cesárea electiva y parto vaginal en cesareadas previas: Comparación de complicaciones maternas neonatales. Rev. Peruana de Ginecología y Obstetricia [Internet] [Fecha de consulta: 2016]; 56: 232 -237.URL disponible en: http://www.redalyc.org/articulo.oa?id=32342819701 1

11. Rodríguez Iglesias Guillermo, León Cid Iraisa, Segura Fernández Anadys, Atienza Barzaga Alba Marina. Vaginal delivery in patients underwent a prior cesarean operation. Rev Cub Med Mil [Internet]. 2011 Dic [citado 2020 Ene 26] ;40( 3-4 ): 218-226. Disponible en: http://scielo.sld.cu/scielo.php?script=sci_arttext\&pid $=$ S0138-65572011000300003\&lng $=$ es.

12. Huertas-Huertas E, Pocco Hinostroza D. Prueba de trabajo de parto en cesareada anterior. Revista Peruana de Ginecolgía y Obstetricia. 2010; 56 (4): 284-285.

http://www.spog.org.pe/web/revista/index.php/RPG O/article/view/212/191

13. Marca Ticona D. Factores asociados con el éxito de la prueba de trabajo de parto en mujeres con cesárea previa en el Hospital Nacional Edgardo Rebagliatti Martins en el año 2017. [Tesis para optar el Título de Médico Cirujano]. [Tacna] : Universidad Jorge Basadre Grohoman ; 2018 P 16. [citado 14 de enero de 2020]. disponible en: ,

http://www.tesis.unjbg.edu.pe/bitstream/handle/UNJ BG/3637/1534_2019_marca_ticona_df_facs_medicina. pdf? sequence $=1$ \&isAllowed $=y$

14. Bujold E, Hammoud AO, Hendler I, Berman S, Blackwell SC, Duperron L, et al., Trial of labor in patients with a previous cesarean section: Does maternal age influence the outcome?.Am J Obstet Gynecol. 2004; 190: 1113-1118.

15. Coassolo KM. Macones G. Seguridad y eficacia del parto vaginal después de una cesárea a las 40 o más semanas de gestación. Universidad de Pensilvania en Filadelfia, EEUU. Obstet Gynecol 2003; 156(1): 57-60

16. Espino Felipa E. Complicaciones materno neonatales en pacientes con parto vaginal luego de cesárea previa en el Hospital María Auxiliadora en el año 2016. (Tesis para optar por el Título profesional de Licenciada en Obstetricia).Universidad Norbert Wiener 2017.

http://repositorio.uwiener.edu.pe/bitstream/handle/1 23456789/1021/TITULO\%20-\%20Espino\%20Felipa\%2 C\%20Elda\%20Johanna.pdf?sequence =1\&isAllowed = $\mathrm{y}$

17. Ricci A. Paolo, Perucca P. Ernesto, Altamirano A. Roberto. Nacimiento por parto vaginal después de una cesárea. Prueba de trabajo de parto versus cesárea electiva a repetición: rotura uterina, una complicación posible. Rev. Chil. Obstet. Ginecol. [internet]. 2003 [citado 2020 ene 15]; 68( 6 ): 523-528. disponible en: https://scielo.conicyt.cl/scielo.php?script=sci_arttext

\&pid=S0717-75262003000600013 
18. Gil Clares Dayana. Parto Vaginal en gestantes con cesárea previa: Hospital Augusto Hernández Mendoza de Ica, periodo de enero a diciembre del 2014. [Tesis para optar el Título de Médico Cirujano] Tacna-Perú. Universidad Jorge Basadre Grohmann 2015. http://repositorio.unjbg.edu.pe/bitstream/handle/UNJ BG/2107/537_2015_gil_clares_dc_facs_medicina_huma na.pdf? sequence $=1$ \&isAllowed $=y$

19. Marca Ticona Diego. Factores asociados con el éxito de prueba de trabajo de parto en mujeres con cesárea previa en el Hospital Edgardo Reblagliatti Martins en el año 2017. [Tesis para optar el Título de Médico Cirujano] Tacna-Perú. Universidad Jorge Basadre Grohmann 2019.

http://www.tesis.unjbg.edu.pe/bitstream/handle/UNJB G/3637/1534_2019_marca_ticona_df_facs_medicina.pd $\mathrm{f}$ ? sequence $=1$ \&isAllowed $=\mathrm{y}$

20. Leal-Mateos Manrique, Giacomin-Carmiol Loretta, Moya-Sibaja Rafael Ángel. Resultados obstétricos y neonatales de pacientes secundigestas con antecedente de cesárea, Hospital Dr. Rafael Ángel Calderón Guardia, 2006-2007Obstetric and Neonatal Outcome in Second-Pregnancy Patients with One Prior Cesarean Section at a Costarican Hospital. Acta méd. costarric [Internet]. 2010 Mar [cited 2020 Jan 31] ; 52( 1 ): 30-34. Available from: http://www.scielo.sa.cr/scielo.php?script=sci_arttext\&p $\mathrm{id}=$ S0001-60022010000100007\&lng=en.

21. Rodríguez Iglesias Guillermo, León Cid Iraisa, Segura Fernández Anadys, Atienza Barzaga Alba Marina. Parto vaginal en pacientes con cesárea anterior. Rev Cub Med Mil [Internet]. 2011 Dic [citado 2020 Ene 31] ; 40 ( 3-4 ): 218-226. Disponible en: http://scielo.sld.cu/scielo.php?script=sci_arttext\&pid= S0138-65572011000300003\&lng=es
22. Vargas Juscamaita Anny Tatiana, Lévano Castro José Antonio, Lazo Porras María de los Ángeles. Parto vaginal después de una cesárea: aplicando un puntaje al momento del ingreso en un hospital. Rev. peru. ginecol. obstet. [Internet]. 2013 Oct [citado 2020 Ene 31] ; 59( 4 ): 261-266. Disponible en: http://www.scielo.org.pe/scielo.php?script=sci_arttex t\&pid=S2304-51322013000400005\&lng=es.

23. Lindo Pérez, Misael et al. Parto vaginal en cesareada anterior: Experiencia de 4 años en un hospital de seguridad social. Revista Peruana de Ginecología y Obstetricia, [S.I.], v. 50, n. 4, p. 217-222, mayo 2015. ISSN 2304-5132. Disponible en: <http://www.spog.org.pe/web/revista/index.php/RP GO/article/view/420/388>. Fecha de acceso: 01 feb. 2020 doi:https://doi.org/10.31403/rpgo.v50i420.

24. Tamayo Barrera Katia. Caracteristicas clinicas y factores asociados de parto vaginal despues de una cesarea previa en el Hospital Cayetano Heredia en el servicio de obstetricia durante el periodo enero 2017 - diciembre 2019. [Trabajo académico para optar el Título de Médico Cirujano] Lima-Perú. Universidad Peruana Cayetano Heredia 2019.

http://repositorio.upch.edu.pe/bitstream/handle/upc h/7061/Caracteristicas_TamayoBarrera_Katia.pdf?seq uence $=1$ \&isAllowed $=\mathrm{y}$

25. Pérez Olazo M. Riesgo materno perinatal del parto vaginal versus cesárea en gestantes con cesárea previa. (Tesis Para optar el Título profesional de Médico Cirujano) Lima-Perú. Universidad de San Martín de Porres 2015.

http://www.repositorioacademico.usmp.edu.pe/bitstr eam/usmp/1853/1/perez_om.pdf

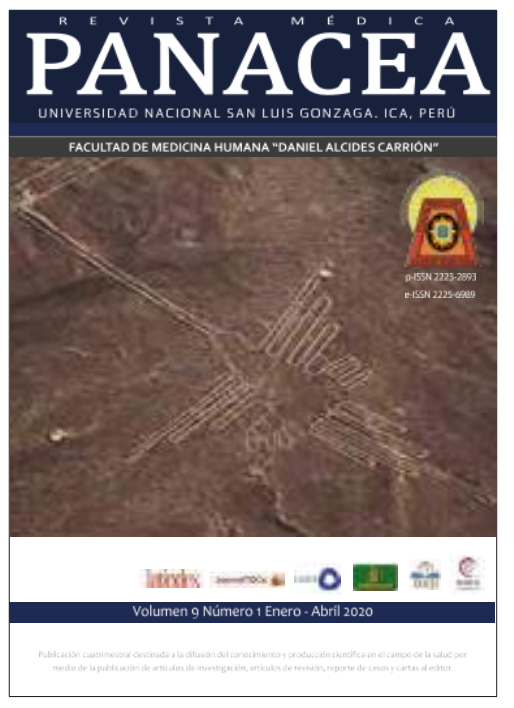

